\title{
THE DEFINITION OF LIE DERIVATIVE
}

\author{
by T. J. WILLMORE
}

(Received 2nd May 1959)

1. The differential operation known as Lie derivation was introduced by $W$. Slebodzinski in 1931, and since then it has been used by numerous investigators in applications in pure and applied mathematics and also in physics. A recent monograph by Kentaro Yano (2) devoted to the theory and application of Lie derivatives gives some idea of the wide range of its uses. However, in this monograph, as indeed in other treatments of the subject, the Lie derivative of a tensor field is defined by means of a formula involving partial derivatives of the given tensor field. It is then proved that the Lie derivative is a differential invariant, i.e. it is independent of a transformation from one allowable coordinate system to another. Sometimes some geometrical motivation is given in explanation of the formula, but this is seldom very satisfying.

The treatment of Lie derivatives given in this note is both natural and simple, and because the derivative is defined without reference to a coordinate system the problem of proving invariance over a transformation of coordinates does not arise. It seems unlikely that the treatment given here can be new, though the author has not come across a similar treatment in the general literature $f$ and it is not to be found in the monograph by Yano.

\section{The Classical Definition}

Let $X$ be a given vector field defined over a differentiable manifold $M$. Let $T$ be a tensor field of type $(p, q)$ (i.e. contravariant of order $p$ and covariant of order $q$ ) defined over $M$. Then the classical definition of the Lie derivative of the tensor field $T$ with respect to the vector field $X$ is the tensor field $L T$ of type $(p, q)$ with components

$$
\begin{aligned}
\underset{X}{(L T)})_{j_{1} \ldots j_{q}}^{i_{1} \ldots i_{p}} & =X^{l} \partial_{l} T_{j_{1} \ldots j_{q}}^{i_{1} \ldots i_{p}}-T_{j_{1} j_{2} \ldots j_{q}}^{i_{2} \ldots i_{p}} \partial_{s} X^{i_{1}} \\
& -T_{j_{1} j_{2} \ldots j_{q}}^{i_{1} s i_{3} \ldots i_{p}} \partial_{s} X^{i_{2}}-\ldots \\
& +T_{s j_{2} \ldots j_{q}}^{i_{1} \ldots i_{p}} \partial_{j_{1}} X^{s}+T_{j_{1} s \ldots j_{q}}^{i_{1} \ldots i_{j_{j}}} \partial_{j_{2}} X^{s}+\ldots
\end{aligned}
$$

That the right-hand member is an invariant may be verified by expressing the partial derivative in terms of covariant derivatives with respect to an arbitrary symmetric connexion, when it is found that the terms involving the connexion coefficients cancel. Thus the partial derivatives can in fact be replaced by covariant derivatives with respect to an arbitrary symmetric connexion.

\section{Extension of Operators}

Before giving our alternative definition of Lie derivation, it is convenient

$\dagger$ See, however, A. Nijenhuis, Abstract 542-200, Notices Amer. Math. Soc. 6, 1958, p. 327. [Added in proof.] 
to consider a general theorem concerned with the extension of differential operators.

Theorem. Let $D$ be a differential operator which maps contravariant vector fields and scalar fields into tensor fields such that

(i) $D$ is the linear over the real numbers, i.e.

$$
D(a X+b Y)=a D X+b D Y
$$

for any vector fields $X, Y$ and any real numbers $a, b$;

$$
D(f Y)=f D Y+(D f) \otimes Y,
$$

for any scalar $f$ and any vector field $Y$.

Then the operator $D$ can be extended in a unique manner to operate on tensor fields of arbitrary type $(p, q)$ in such a way that

(iii) it is linear over the real numbers, i.e.

$$
D(a S+b T)=a D S+b D T,
$$

for any tensors $S, T$ of the same type, and for any real numbers $a, b$;

(iv) the Leibnitz law of derivation of a product is satisfied, i.e.

$$
D(S \otimes T)=(D S) \otimes T+S \otimes(D T),
$$

for all tensors $S, T$;

(v) the operator commutes with the process of contraction; alternatively

$$
D I=0,
$$

where I is the Kronecker tensor.

The proof of the theorem is straightforward. For example, to extend the domain of the operator to tensor fields of type $(2,0)$, it is sufficient to note that any tensor $T$ of this type can be expressed as a linear combination of decomposable tensors, i.e.

$$
T=\sum_{k=1}^{m} f_{k} X^{k} \otimes Y^{k}
$$

where $f_{k}$ are scalars and $X^{k}, Y^{k}$ vector fields. Then by using (ii) and (iv), $D T$ is determined.

If $\mu$ is a given covariant vector field and $\lambda$ an arbitrary contravariant vector field, it follows that

which determines $D \mu$.

$$
<\lambda, D \mu>=D(<\lambda, \mu>)-<D \lambda, \mu>
$$

The extension of $D$ to a tensor field of type $(p, q)$ can now be obtained by expressing the tensor as a linear combination of tensor products of contravariant and covariant vector fields.

\section{Application to Lie Derivatives}

Let $X$ be a given vector field over $M$. If $Y$ is another vector field over $M$, it is well-known that $X$ and $Y$ determine a third vector field denoted by $[X, Y]$, 
defined by

$$
[X, Y]=X Y-Y X \text {. }
$$

The vector field $[X, Y]$ is the classical Poisson bracket or Lie bracket. The mapping

$$
Y \rightarrow[X, Y]
$$

will be denoted by $D$.

The vector field $X$ operates on a scalar field $f$ according to the usual law,

$$
f \rightarrow X f \text {. }
$$

The mapping (4.3) will also be denoted by $D$.

From (4.2) it follows that

$$
D(f Y)=[X, f Y]=X(f Y)-f(Y X) .
$$

If we use the fact that a tangent vector is a derivation, i.e. at any point $P$,

$$
X(f g)=X(f) . g(P)+f(P) . X(g),
$$

(4.4) becomes

$$
\begin{aligned}
D(f Y) & =f(X Y-Y X)+(X f) \otimes Y \\
& =f[X, Y]+X f \otimes Y, \\
\text { i.e. } \quad D(f Y) & =f D Y+D f \otimes Y,
\end{aligned}
$$

so that $D$ satisfies condition (ii) of $\S 3$. It is trivial that $D$ satisfies condition (i) of $\S 3$. It follows from the theorem of $\S 3$ that the operator $D$ can be extended in a unique manner to operate on an arbitrary tensor field of type $(p, q)$ so that conditions (iii), (iv) and (v) are satisfied. This extended operator is defined to be the Lie derivative.

To establish that this definition is consistent with the classical definition given in $\S 2$, it is sufficient to note that the operator defined in $\S 2$ satisfies conditions (i), (ii), (iii), (iv), (v). Moreover, the two operators agree when operating on scalar fields and vector fields, and so by the uniqueness of the extension, it follows that the two operators must agree on tensor fields of type $(p, q)$.

The theorem of $\S 3$ can be used to give coordinate-free definitions of other differential operators, including the operation of torsional derivation (1).

\section{REFERENCES}

(1) T. J. Willmore, Generalised torsional derivation, Atti. Accad. Naz. Lincei, 26 (1959), 649-653.

(2) Kentaro, Yano, The Theory of Lie Derivatives and its Applications, NorthHolland Publishing Co., Groningen (1957).

Mathematical Institute UNIVERSITY OF LIVERPOOL 